\title{
Antibodies against swine influenza virus neutralize the pandemic influenza virus A/H1N1
}

\author{
MASAYA TSUKAMOTO ${ }^{1}$, SATOSHI HIROI ${ }^{2}$, KAZUHIDE ADACHI ${ }^{1}$, HIDENORI KATO ${ }^{1}$, \\ MARIE INAI $^{1}$, ITSURO KONISHI ${ }^{3}$, MASARU TANAKA ${ }^{3}$, RYOUHEI YAMAMOTO ${ }^{3}$, \\ MASAKI SAWA $^{3}$, EKOWATI HANDHARYANI ${ }^{4}$ and YASUHIRO TSUKAMOTO ${ }^{1}$ \\ ${ }^{1}$ Department of Animal Hygiene, Graduate School of Environmental and Biological Sciences, \\ Kyoto Prefecture University, Kyoto 606-8522; ${ }^{2}$ Department of Virology, Osaka Prefectural Institute \\ of Public Health, Osaka 537-0025; ${ }^{3}$ Ostrich Breeding Farm 'Ostrich-Kobe', Hyogo 651-2321, Japan; \\ ${ }^{4}$ Faculty of Veterinary Medicine, Bogor Agriculture University, Bogor 16680, Indonesia
}

Received September 13, 2010; Accepted December 14, 2010

DOI: $10.3892 / \mathrm{mmr} .2011 .410$

\begin{abstract}
The most effective method for the prevention of influenza infection would be prophylaxis with a safe and effective vaccine and anti-viral materials. After vaccination, neutralizing antibodies are generated by plasma cells following various immune responses, thus resulting in protection against an infectious agent expressing the same antigens. However, in the case of novel or unknown pathogens, the onset of immune responses is occasionally delayed, thus resulting in considerable morbidity and mortality. Antibodies are therefore considered to play an important role in preventing infectious diseases. Furthermore, antibodies are used for additional purposes, including diagnosis and immunotherapy. In the beginning of spring 2009, an outbreak of influenza in North America was caused by a novel strain of influenza virus, designated pandemic influenza A/H1N1 2009. Initially, most people had low immunity against this pathogen, resulting in the worldwide spread of the infection to produce a so-called 'pandemic'. We herein report the generation of 'immunoglobulin yolk (IgY)' neutralizing antibodies against the pandemic influenza virus A/H1N1 from ostrich eggs immunized with a swine influenza virus vaccine strain. Using this simple method, a large amount of specific antibody against the influenza virus was produced by one female ostrich. An enzyme-linked immunosorbant assay and immunocytochemistry indicated that the IgY from the immunized ostrich eggs possessed strong cross-reactivity to the pandemic influenza
\end{abstract}

Correspondence to: Professor Yasuhiro Tsukamoto, Department of Animal Hygiene, Graduate School of Biology and Environmental Sciences, Kyoto Prefecture University, 1-5 Nakaragicho, Shimogamo, Kyoto 606-8522, Japan

E-mail: ytsuka@kpu.ac.jp

Key words: influenza virus, pandemic, H1N1, antibody, ostrich, egg virus $\mathrm{A} / \mathrm{H} 1 \mathrm{~N} 12009$, as well as to the swine influenza virus. Moreover, the hemaggregation activities of the erythrocytes induced by pandemic influenza $\mathrm{A} / \mathrm{H} 1 \mathrm{~N} 1$ virus were inhibited by the ostrich antibodies generated by swine virus immunization. In addition, the cytopathological effects on MDCK cells of infection with pandemic virus were clearly inhibited in co-cultures with the antibodies, indicating the neutralizing of viral infectivity in the cells. In conclusion, we have succeeded in the mass production of neutralizing antibodies against pandemic influenza virus A/H1N1 2009 using ostrich eggs immunized with swine influenza virus antigens. This enables the cost-effective production of effective antibodies, which could be applied to facial masks and air-conditioning filters in order to prevent populations from acquiring pandemic influenza virus $\mathrm{A} / \mathrm{H} 1 \mathrm{~N} 1$.

\section{Introduction}

Influenza is recognized as a zoonotic disease, with the most commonly affected animals being humans, pigs, horses and species of aquatic birds (1-4). Influenza viruses belong to the family Orthomyxoviridae and are divided into three types, A, B and C. A-type viruses are responsible for major disease problems in humans, as well as in avian species, and are further classified into subtypes on the basis of their antigenic properties, including the type of hemagglutinin (HA) and neuraminidase (NA) on the viral particle. Two A types (H1N1 and $\mathrm{H} 3 \mathrm{~N} 2$ ) and various B types are presently circulating in the human population. Ten to fifteen percent of people worldwide contract influenza annually, with rates soaring as high as 50\% during major epidemics.

In April 2009, it became apparent to public health officials in Mexico City that an outbreak of influenza was in progress late in the influenza season. The virus from patients was determined to be a novel strain of influenza A of the H1N1 serotype. Detailed genetic examinations indicated that the virus was a novel reassortant containing genetic elements of influenza viruses found in swine, birds and humans (5). Less than 1 month later, thousands of 
probable cases of infection by this novel virus, designated Influenza H1N1 2009, had been identified, and many deaths had occurred in Mexico. Sporadic cases, mostly associated with travel to Mexico, were subsequently noted in several other countries, including the US, Canada and various countries in Europe, Asia and Africa. The World Health Organization (WHO) began to declare ever higher stages on its pandemic scale, designating the novel influenza H1N1 2009 a potential threat to the worldwide health. Infection with this pandemic influenza $\mathrm{A} / \mathrm{H} 1 \mathrm{~N} 1$ virus spread across the world in 2009 and 2010.

Vaccination has been a mainstay of influenza prevention, with annual vaccination recommended for adults and children at a high risk. Efforts to interrupt person-to-person transmission are also important $(6,7)$. The CDC in the US has recommended that health-care facilities implement a universal respiratory hygiene strategy, including providing masks or facial tissues in waiting rooms to people with respiratory symptoms (4). The development of a high-grade mask with new prevention mechanisms appears necessary in order to curb the spread of such a disease (8).

There is an increasing use of antibodies for research, diagnosis and therapeutic purposes. However, antibodies from experimental mammals, including mouse and rabbit, are not adapted for industrial use, due to their high production cost. Lately, the avian egg has proven to be an attractive source for the non-invasive production of antibodies with application in research, diagnosis and immunotherapy (9-11). In addition, the production of avian antibodies offers numerous advantages over mammalian antibodies regarding their specificity for antigens, production cost and uses (10). The predominant class of immunoglobulin in birds is immunoglobulin yolk (IgY), which is transferred from the serum to the yolk to confer passive immunity to the embryo (12). Although the $\mathrm{IgY}$ and $\mathrm{IgG}$ of mammals are functionally equivalent, they differ with respect to certain physicochemical properties (13). Recently, our research group developed a convenient method for the mass-production of antibodies using the female ostrich (Struthio camelus) $(14,15)$. The ostrich grows up to $250 \mathrm{~cm}$ in height and $160 \mathrm{~kg}$ in weight, and their life span is approximately 60 years. The ostrich eggs weigh approximately $1.5 \mathrm{~kg}$ and are 30 -fold bigger than chicken eggs. Ostriches lay 100 eggs every year. We purify 2-4 g of IgY per ostrich egg. Accordingly, about $400 \mathrm{~g}$ of IgY is obtained from only one ostrich in the course of a year. Recent developments in breeding have permitted low-cost egg production from the ostrich, since they are fed with factory waste from beansprout production. Accordingly, we strongly believe that the ostrich egg might provide an excellent source of antibody for industrial purposes.

In the present study, we attempted the production of neutralizing antibodies against the pandemic influenza viruse A/H1N1 2009 from ostrich eggs. Since work with this novel virus had to be performed at a high bio-safety level, and a system using cultured cells or embryos had yet to be developed, we had difficulties obtaining antigens for immunization into the animals. Pandemic influenza A/H1N1 2009 is a novel virus, but belongs to a serotype of $\mathrm{A} / \mathrm{H} 1 \mathrm{~N} 1$, which includes seasonal influenza virus in humans. Furthermore, this virus is genetically similar to the swine influenza $\mathrm{A} / \mathrm{H} 1 \mathrm{~N} 1$ virus of
Japanese origin. Vaccine strains of swine influenza viruses are adapted to growth in either cultured cells or chicken eggs, and are easy to handle. Accordingly, these influenza viruses were considered suitable antigens for immunizing the ostrich to obtain neutralizing antibodies against the pandemic virus.

We herein reveal that a high amount of neutralizing antibody against the pandemic influenza virus A/H1N1 2009 was generated by the ostrich using a simple and economical method involving immunization with a similar virus. Our strategy may lead to the development of a novel mask or filter impregnated with antibody, which protects against influenza infections.

\section{Materials and methods}

Virus. A swine influenza virus A/H1N1 and clinical pandemic influenza viruse A/H1N1 2009 were used in this study.

The swine influenza virus, A/swine/Kyoto 3/79 (H1N1), was a vaccine strain for swine infection. This virus was a low-pathogenic strain from domestic pigs. A small volume of viral fluid was injected into embryonic chicken eggs at 10 days post incubation, and the virus was collected 2 days later from chorioallantoic fluid and used for further studies. Nine strains of pandemic influenza virus H1N1 were cloned from the patients in Osaka Prefecture Japan and numbered as A/Osaka/47/2009 (H1N1) pdm, A/Osaka/61/2009 (H1N1) pdm, A/Osaka/63/2009 (H1N1) pdm, A/Osaka/159/2009 (H1N1) pdm, A/Osaka/163/2009 (H1N1) pdm, A/Osaka/ 2037/2009 (H1N1)pdm, A/Osaka/2040/2009 (H1N1) pdm, A/Osaka/2048/2009 (H1N1) pdm and A/Osaka/2051/2009 (H1N1) pdm. Since the primary viruses in nasal swab samples from patients did not grow well under in vitro experimental conditions, several passages on MDCK cells were performed, leading to an adaptation in cultured cells. Three strains, A/Osaka/2040/2009 (H1N1) pdm, A/Osaka/47/2009 (H1N1) pdm and A/Osaka/2048/2009 (H1N1) pdm, were employed for neutralizing assays due to their adapted growth in culture cells.

Generation of ostrich antibody by immunization with swine influenza virus. An inactivated vaccine including 400 CCA units of A/swine/Kyoto 3/79 (H1N1) (Kyoto-Biken Laboratories Inc., Japan) was emulsified in Freund's complete adjuvant and injected intra-muscularly to the lumbar region at multiple sites of a laying ostrich. Boosters were administered every other week with the antigen in Freund's incomplete adjuvant. The experiments were approved by the animal care committee of Kyoto Prefecture University. The eggs were collected weekly from the initial immunization day. The yolk was separated from the albumin and the volume of the yolk was recorded and stored at $-20^{\circ} \mathrm{C}$. Serum samples were also collected every week and stored at $-20^{\circ} \mathrm{C}$ until analysis. The yolk was separated from the albumin of the eggs and diluted 5-fold with TBS buffer [0.02 M Tris- $\mathrm{HCl}(\mathrm{pH} 7.5), 0.15 \mathrm{M}$ $\mathrm{NaCl}$ ], added at a ratio of 1:10 to $30 \%$ dextran sulfate in TBS, diluted 2- to 3-fold with $2.5 \mathrm{M} \mathrm{CaCl}_{2}$ in TBS, then stored at $4^{\circ} \mathrm{C}$ for at least $4 \mathrm{~h}$. The supernatant containing the IgY was collected by centrifugation $\left(10,000 \mathrm{x}\right.$ g at $4^{\circ} \mathrm{C}$ for $\left.15 \mathrm{~min}\right)$ and precipitated with $45 \%$ saturated ammonium sulfate. The solution was centrifuged again at $10,000 \mathrm{xg}$ at $4^{\circ} \mathrm{C}$ for $15 \mathrm{~min}$. The 
precipitate was then re-dissolved in TBS and dialyzed against PBS. Finally, the purified antibody solutions were verified by 10\% SDS-PAGE under non-reducing conditions and stained with Coomassie Brilliant Blue.

Enzyme-linked immunosorbant assay. Each well of a polystyrene enzyme-linked immunosorbant assay (ELISA) plate (Sumitomo Bakelite, Japan) was coated with $0.2 \mu \mathrm{g}$ of A/ swine/Kyoto 3/79 (H1N1) or hemagglutin (HA) antigens from pandemic influenza virus A/H1N1 (2009) (Protein Science, USA) in phosphate-buffered saline (PBS), then the plate was incubated overnight at $4^{\circ} \mathrm{C}$. Each of the following incubation steps was preceded by washing of the wells twice with PBS containing $0.05 \%$ Tween-20. The wells were blocked from non-specific binding by the addition of a commercial blocking buffer (DS Pharma Biomedical, Japan) and were incubated at $37^{\circ} \mathrm{C}$ for $2 \mathrm{~h}$. Serial dilutions of purified $\mathrm{IgY}$ were added vertically to the wells, followed by incubation at $37^{\circ} \mathrm{C}$ for $1 \mathrm{~h}$. Subsequently, HRP-conjugated secondary antibody (rabbit IgG against ostrich IgY) diluted (1:5,000) in PBS was dispensed into each well. The plate was incubated for $1 \mathrm{~h}$ at $37^{\circ} \mathrm{C}$. Later, a substrate buffer containing TMB (Sumitomo Bakelite) was added to each well, and the samples were incubated at $37^{\circ} \mathrm{C}$ for $15 \mathrm{~min}$. The reaction was terminated by the addition of a stopping reagent (1.25 $\mathrm{M}$ sulfuric acid). The absorbance was recorded at $450 \mathrm{~nm}$ using the ELISA plate reader.

Immunocytochemistry for pandemic influenza virus A/H1MI using ostrich antibodies. MDCK cells seeded in the wells of 96-well plates were infected with swine A/H1N1 and clinical strains of pandemic influenza virus $\mathrm{A} / \mathrm{H} 1 \mathrm{~N} 1$ for $2-5$ days at $35^{\circ} \mathrm{C}$. The cultures were fixed with $10 \%$ buffered formalin for immunocytochemistry. The cells were directly incubated with ostrich IgY conjugated with FITC (Dojin Laboratories, Japan) for $1 \mathrm{~h}$ at $37^{\circ} \mathrm{C}$ following washes in PBS. Finally, the specific signal was observed under a fluorescence microscope.

Hemagglutination test. Whole blood from guinea pigs was washed with PBS, and the erythrocytes were prepared after centrifugation. Serial 2-fold dilutions of clinical strains of pandemic influenza virus $\mathrm{A} / \mathrm{H} 1 \mathrm{~N} 1$ were mixed with guinea pig erythrocyte solutions in clear 96-well micro test polystyrene assay plates (Becton Dickinson, USA). After $45 \mathrm{~min}$ at room temperature, the hemaggregation activity was observed and the hemagglutination titers of the virus were measured. The highest dilutions, indicating clear hemaggregation, were scored as HA titers.

Hemagglutination inhibition test. Serial dilutions of ostrich IgY were mixed with 8-HA units of pandemic influenza virus [A/Osaka/2040/2009 (H1N1) pdm, A/Osaka/47/2009 (H1N1) pdm and A/Osaka/2048/2009 (H1N1) pdm] in clear 96-well micro test polystyrene assay plates. After a 30-min incubation at room temperature, guinea pig erythrocytes were added, pipetted gently and then incubated for another $45 \mathrm{~min}$ at room temperature. The hemaggregations in each well were observed and the hemagglutination inhibition (HI) titers were scored as 'the highest dilution of pre-immune IgY indicating clear inhibition on hemaggregations' vs. 'the highest dilution of immune IgY indicating clear inhibition on hemaggregations'.

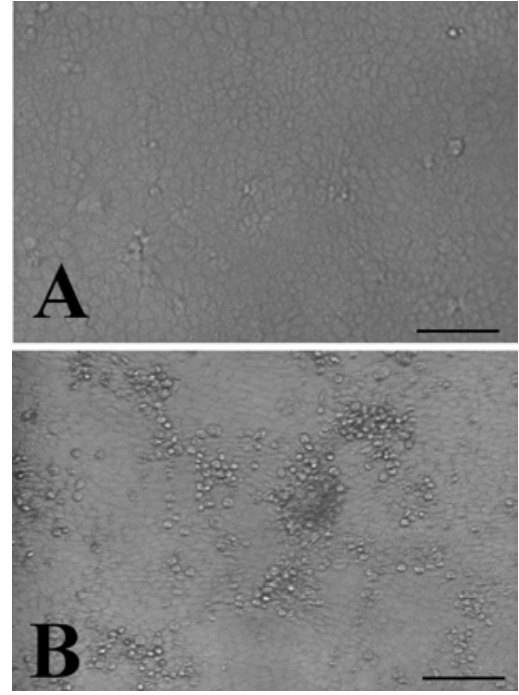

Figure 1. Infection of the pandemic influenza virus A/H1N1 in MDCK cells Monolayers of MDCK cells were inoculated with the pandemic influenza virus $\mathrm{A} / \mathrm{H} 1 \mathrm{~N} 1$ following trypsin digestion; cells were incubated for 5 days. Uninfected MDCK cells show clear monolayers (A). By contrast, the cells infected with a strain of pandemic influenza A/Osaka/2040/2009 (H1N1) show typical cytopathic effects (CPE): numerous cells are rounding and floating (B). Bars, $100 \mu \mathrm{m}$

Accordingly, a higher ratio indicates a stronger inhibitory activity of the antibody against the influenza virus.

Neutralizing assays for pandemic influenza virus infections. Serial dilutions of ostrich IgY were mixed with A/swine/ Kyoto/3/79 (H1N1) and with above strains of pandemic influenza virus $\mathrm{A} / \mathrm{H} 1 \mathrm{~N} 1$ at $100 \mathrm{TCID}_{50}$ and incubated for $1 \mathrm{~h}$ at $37^{\circ} \mathrm{C}$. Thereafter, these viral fluids were adsorbed onto MDCK monolayers for $1 \mathrm{~h}$ at $35^{\circ} \mathrm{C}$ and then incubated in GIT media (Gibco) containing low trypsin at $35^{\circ} \mathrm{C}$. At 5 days post-incubation, the cultures were inspected for the cytopathic effect (CPE): the neutralizing titer, expressed as the reciprocal of IgY dilution at which virus growth is $50 \%$ inhibited, was calculated by the number of virus negative wells and the IgY dilution according to the report by Reed and Muench (16).

\section{Results}

Adaptation of clinical strains of pandemic influenza virus A/H1N1 to be grown in MDCK cells. Following considerable passages on MDCK cells, a total of nine strains of pandemic influenza virus $\mathrm{A} / \mathrm{H} 1 \mathrm{~N} 1$ from patients appeared to grow easily under experimental conditions. In addition, these viral strains induced obvious CPEs in MDCK cells after infection (Fig. 1). Among these viruses, three strains [A/Osaka/2040/2009 (H1N1) pdm, A/Osaka/47/2009 (H1N1) pdm and A/Osaka/2048/2009 (H1N1) pdm] with rapid growth in cells were employed in neutralizing assays.

Generation of ostrich antibodies immunized with swine influenza virus $A / H 1 N 1$ and its cross-reactivity to the pandemic influenza virus $A / H 1 N 1$. The ostrich $\operatorname{IgY}$ from the ostriches immunized with swine influenza virus was purified from the yolk and visualized by SDS-PAGE. The molecular weight of 
Table I. Generation of ostrich antibodies by swine influenza virus immunization.

Serum or antibody generated

from immunized female ostrich
Antibody titer against indicated

influenza virus HA antigens

Swine A/H1N1

Pandemic A/H1N1

Serum

6400

3200

Antibody (IgY)

6400

12800

The antibody titer against swine influenza and pandemic influenza viruses was estimated by ELISA. At 12 weeks post-immunization, the ELISA titers against A/swine/Kyoto/3/79 (H1N1) were clearly increased in both ostrich serum and egg yolk (IgY). This indicates that the serum antibody was transferred into the yolk. In addition, reactivity against hemagglutination from pandemic influenza virus A/H1N1 was also increased in the serum and antibody (IgY). This indicates that the antibody is cross-reactive to the pandemic influenza virus A/H1N1. The antibody titers were defined as the reciprocal of the highest dilution that produced an ELISA signal twice as intense as the signal from equivalently diluted preimmune serum or yolk.

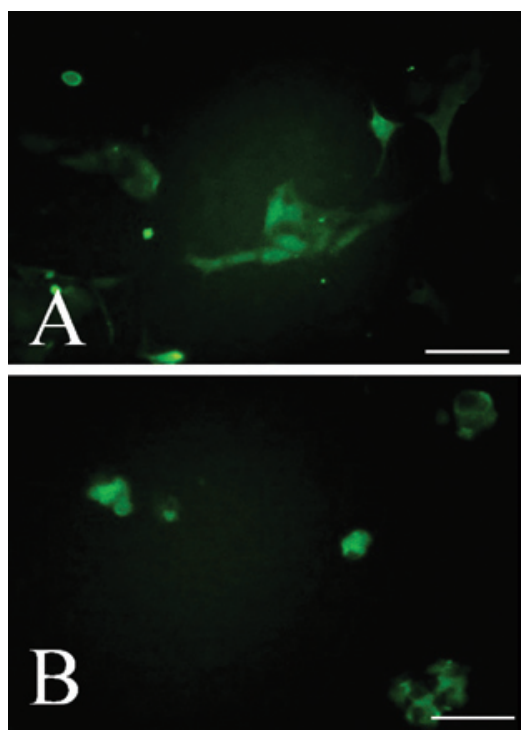

Figure 2. Immunocytochemistry of cells infected with the pandemic influenza virus A/H1N1 using ostrich IgY generated by the swine influenza virus immunization. MDCK cells were infected with a swine influenza (A) and the strains of pandemic influenza viruse $\mathrm{A} / \mathrm{H} 1 \mathrm{~N} 1$ (B), and reacted with FITC-conjugated ostrich IgY generated by immunization with the swine influenza viruses. Positive reactions were seen in the pandemic influenza A/Osaka/2040/2009 (H1N1)-infected MDCK cells, as well as in the cells infected by A/swine/Kyoto/3/79 (H1N1). Therefore, the ostrich IgY crossreacts to both swine and pandemic influenza viruses. Scale bars, $100 \mu \mathrm{m}$.

the ostrich IgY was around $200 \mathrm{kDa}$ (data not shown). The immunoreactivities of antisera and IgY against swine influenza virus and pandemic virus were estimated by ELISA. The titers for each antigen were increased dramatically in both the sera and yolk after the initial immunization (Table I). These findings indicate that reactive antibodies were generated and transmitted into the bloodstream, and were also successfully transferred into the yolk in immunized female birds. In addition, it appears that the IgY had cross-reactivity to the pandemic influenza viral antigens, as well as to a swine virus. At the hyper-immune stage from 12 weeks, it was possible to purify 2-4 g of IgY from one egg, thus indicating that we would be able to collect $400 \mathrm{~g}$ of IgY against the pandemic influenza virus from one female ostrich in 1 year.
Table II. Inhibitory activity of ostrich IgY on hemaggregations by pandemic influenza virus $\mathrm{A} / \mathrm{H} 1 \mathrm{~N} 1$.

Virus HI titer of ostrich IgY

A/swine/Kyoto/3/79 (H1N1) 32

A/Osaka/47/2009 (H1N1) pdm 64

A/Osaka/2040/2009 (H1N1) pdm

A/Osaka/2048/2009 (H1N1) pdm

Guinea pig erythrocytes were reacted with $\mathrm{A} / \mathrm{swine} / \mathrm{Kyoto} / 3 / 79$ (H1N1) and three clinical strains of pandemic influenza virus A/H1N1 (A/Osaka/2040/2009, A/Osaka/47/2009 and A/Osaka/2048/2009) after incubation with IgY. The activity of IgY against each of the viruses was represented as the hemagglutination inhibition (HI) titer ratio: the highest dilution of preimmune IgY indicating clear inhibition on hemaggregation vs. the highest dilution of immune IgY indicating clear inhibition on hemaggregation. The higher HI titers indicate a strong inhibitory activity of the antibody against aggregation by the viruses. The ostrich IgY reveals inhibitory activities on hemaggregation by all three strains of pandemic influenza viruses, as well as on the swine virus.

Next, the reactivity of ostrich $\operatorname{IgY}$ was examined by immunocytochemistry. The MDCK cells were infected with the pandemic influenza virus $\mathrm{A} / \mathrm{H} 1 \mathrm{~N} 1$ from patients or with the swine A/H1N1. After a brief fixation with $20 \%$ buffered formalin, the infected cells were stained with FITCconjugated $\operatorname{IgY}$ generated by swine influenza immunization. The cytoplasm of the MDCK cells infected with each influenza virus was strongly labeled with the ostrich IgY generated by swine influenza virus immunization (Fig. 2). By contrast, pre-immune IgY did not react with the cells infected with either swine or pandemic virus (data not shown). These results indicate that the cross-reactivity of IgY between swine influenza and pandemic influenza virus A/H1N1 was specific and was successfully elicited in the ostriches, thereby supporting the ELISA findings.

Inhibition of hemaggregation activities of pandemic influenza virus A/HINI by ostrich IgY. First, the HA activities of the 
Table III. Neutralizing activities of ostrich IgY against the infectivities of swine and pandemic influenza virus A/H1N1.

\begin{tabular}{lcc}
\hline Antibody $(\mathrm{IgY})$ & \multicolumn{2}{c}{ Neutralizing titers $(\mu \mathrm{g} / 25 \mu \mathrm{l})$} \\
\cline { 2 - 3 } & Swine A/H1N1 & Pandemic A/H1N1 \\
\hline Preimmune IgY & $>25.0$ & $>25.0$ \\
Immunized IgY & 0.8 & 1.6 \\
\hline
\end{tabular}

Neutralizing assays were performed using MDCK cells infected with swine and strains of pandemic influenza viruse $\mathrm{A} / \mathrm{H} 1 \mathrm{~N} 1$. The titers are indicated as the mean of the $50 \%$ inhibition on CPE at 5 days post-infection. Infection by both swine and pandemic influenza viruses was inhibited by a small volume of immunized ostrich IgY.

pandemic influenza virus were estimated using erythrocytes, since the viral strains in this study originated from sporadic cases of infection and their characteristics have not yet been clarified. Fortunately, some of the nine strains caused strong aggregations of guinea pig erythrocytes. Accordingly, it was revealed that the clinical pandemic $\mathrm{A} / \mathrm{H} 1 \mathrm{~N} 1$ virus binds to the erythrocytes through their surface HA antigens. The highest dilutions of viral fluids revealing that the hemaggregation was scored as a single HA unit (HAU). The strains [A/ Osaka/2040/2009 (H1N1) pdm, A/Osaka/47/2009 (H1N1) pdm and A/Osaka/2048/2009 (H1N1) pdm] were used for further $\mathrm{HI}$ testing at $8 \mathrm{HAU}$.

Hemaggregations by the swine influenza virus were dramatically inhibited by the ostrich IgY. Notably, the hemaggregation activities of all three clinical strains of the pandemic influenza virus were perturbed by the IgY (Table II).

Neutralizing assays for pandemic influenza virus A/HINI infection. The swine influenza and pandemic influenza virus A/H1N1 were reacted with IgY followed by inoculation into MDCK cells. As shown in Fig. 3 and Table III, the CPEs of MDCK cells infected with either swine or pandemic A/ H1N1 were inhibited by the IgY from swine virus-immunized ostrich. Accordingly, it is clear that this ostrich IgY inhibited the infection of the pandemic influenza virus A/H1N1, as well as the swine influenza virus.

\section{Discussion}

In the present study, the potential for using ostriches for the large-scale and economical production of neutralizing antibodies against pandemic influenza viruses was explored. We focused on the swine influenza virus $\mathrm{A} / \mathrm{H} 1 \mathrm{~N} 1$, since this Japanese virus belongs to the same group as the North American type of swine flu, and was thus closer to the pandemic influenza virus $\mathrm{A} / \mathrm{H} 1 \mathrm{~N} 1$ based on the HA gene sequences, providing a better likelihood of cross-reactivity to different viruses. Female ostriches produced cross-reactive antibodies after immunization with the swine influenza virus, which were transferred into the yolk from the serum. In the hyper-immune stage, up to $4 \mathrm{~g}$ of $\mathrm{IgY}$ could be purified from one yolk, indicating that $400 \mathrm{~g}$ of antibodies could be collected from one female ostrich in 1 year. This suggests that

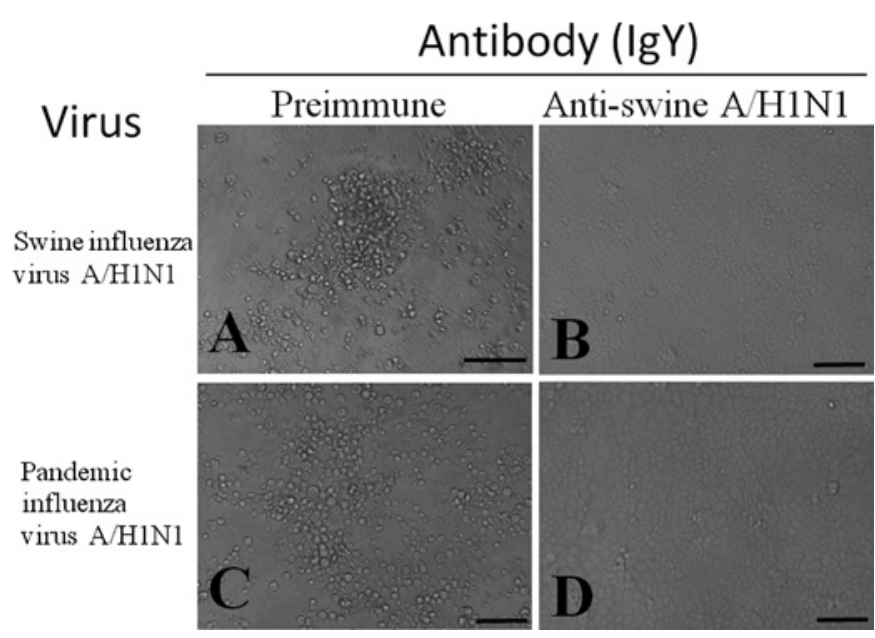

Figure 3. Neutralization of pandemic influenza virus $\mathrm{A} / \mathrm{H} 1 \mathrm{~N} 1$ by ostrich IgY against swine influenza virus. A swine and pandemic influenza virus A/H1N1 were inoculated into MDCK with pre-immune IgY or anti-swine influenza virus IgY. MDCK infected with A/swine/Kyoto/3/79 (H1N1) revealed apparent $\mathrm{CPE}$ in the presence of pre-immune $\mathrm{IgY}$ (A) at 5 days post-infection, whereas the CPE was inhibited by the IgY against swine influenza virus (B). Notably, infection by the pandemic influenza virus A/ Osaka/2040/2009 (H1N1) was clearly inhibited by the IgY (D), but not by the pre-immune IgY (C). Bars, $100 \mu \mathrm{m}$.

anti-pandemic influenza virus antibodies can be produced in large quantities at a relatively low price by using ostriches immunized with the swine influenza virus.

Since HA is essential for viral binding to cells and entrance into host cells, these antigens are widely used in the vaccination against influenza in humans $(17,18)$. As a result, the inhibition of HA antigens by antibodies is useful for protecting against these viral infections. One particularly interesting and potentially important finding of the present study was the observation that ostrich IgY against the swine influenza virus perturbed the hemaggregation activities of the pandemic influenza virus $\mathrm{A} / \mathrm{H} 1 \mathrm{~N} 1$. We speculate that the $\mathrm{HA}$ antigens on the pandemic influenza virus $\mathrm{A} / \mathrm{H} 1 \mathrm{~N} 1$ might be masked by ostrich antibodies, thus resulting in the effective blocking of viral adsorption onto the cells.

To confirm this paradigm, the neutralizing activities of antibodies were assessed using living cells. The infection of pandemic influenza viruses from patients was prevented by the ostrich antibody IgY. The CPE of MDCK cells was dramatically inhibited by the antibodies. Therefore, the antibodies may inhibit the entrance of the pandemic influenza virus A/H1N1 into cells by blocking HA activities, resulting in the escape of cells from the viral infection. Further studies are required to verify the inhibitory potential of ostrich antibodies on other antigens of the pandemic influenza virus, including $\mathrm{M}$ and NA, since these antigens also play a considerable role in viral infections and release.

Recently, various types of facial masks and air-conditioning filters have been used for the prevention of airborn infections. However, the small influenza virus passes through the currentlyused filters, thus resulting in human infection, because the virus is still alive even after drying $(8,19,20)$. It thus appears that a high grade filter employing new prevention mechanisms needs to be developed. We applied the ostrich IgY onto the filters in order to create novel filters resulting in protection 
against the influenza virus by antigen-antibody reactions. A large amount of neutralizing antibodies against the influenza virus were produced in a cost-effective manner, which indicates the potential usage of ostrich antibodies for industrial purposes. We believe that filters impregnated with ostrich antibodies will therefore become a powerful tool for protection against pandemic influenza viruses.

\section{Acknowledgements}

We thank Dr Tetsuo Kase of the Osaka Prefectural Institute of Public Health for providing the pandemic strains of influenza virus A/H1N1. We also thank Dr Yasuhara at Kyoto-Biken Laboratories Inc. for providing the vaccine strain of swine influenza virus. This study was supported in part by a Grantin-Aid for Scientific Research (No. 21380182) from the Ministry of Education, Science, Sports and Culture, Japan.

\section{References}

1. Alexander DJ and Brown IH: Recent zoonoses caused by influenza A viruses. Rev Sci Tech 19: 197-225, 2000.

2. Rimmelzwaan GF and Osterhaus AD: Influenza vaccine: new developments. Curr Opin Pharmacol 1: 491-496, 2001.

3. Normile D: North Korea collaborates to fight bird flu. Science 308: 175, 2005.

4. Center for Disease Control and Prevention (CDC): Experiences with influenza-like illness and attitude regarding influenza prevention. United States, 2003-2004 influenza season. MMWR 53: 1156-1158, 2004.

5. Gallaher WR: Towards a sane and rational approach to management influenza H1N1 2009. Virol J 6: 51-59, 2009.

6. Poland GA: Vaccines against avian influenza - a race against time. N Engl J Med 354: 1411-1413, 2006.

7. Sharpe M, Lynch D, Topham, Major D, Wood J and Loudon P: Protection of mice from $\mathrm{H} 5 \mathrm{~N} 1$ influenza challenge by prophylactic DNA vaccination using particle mediated epidermal delivery. Vaccine 25: 6392-6398, 2007.

8. Wiwanitkit V: N-95 face mask for prevention of bird-flu virus: an appraisal of nanostructure and implication for infectious control. Lung 184: 373-374, 2006
9. Schade R, Pfister C, Halatsch R and Henklein P: Polyclonal IgY antibodies from chicken egg yolk - an alternative to the production of mammalian $\operatorname{IgG}$ type antibodies in rabbits. ATLA 19: 403-419, 1991

10. Schade R, Schniering A and Hlinak A: Polyclonal avian antibodies extracted from egg yolk as an alternative to the production of antibodies in mammals - a review. ALTEX 9: 43-56, 1992.

11. Gross $M$ and Speck J: Avian yolk antibodies in diagnosis and research. Dtsch Tierarztl Wochenschr 103: 417-422, 1996.

12. Larsson A, Balow RM, Lindahl TL and Forsberg PO: Chicken antibodies: taking advantage of evolution-a review. Poult Sci 72: 1807-1812, 1993.

13. Leslie GA and Clem LW: Phylogeny of immunoglobulin structure and function. III. Immunoglobulins of the chicken. J Exp Med 130: 1337-1352, 1969.

14. Adachi K, Hagimori K, Kato H, Fukuda K, Kikuta M and Tsukamoto Y: Potential role of SC1, a cell adhesion molecule, in mammary gland tumors. Mol Med Rep 1: 219-224, 2008.

15. Adachi K, Handharyani E, Sari DK, Takama K, Fukuda K, Dndo I, Yamamoto R, Sawa M, Tanaka M, Konisi I and Tsukamoto Y: Development of neutralizing antibodies against highly pathogenic $\mathrm{H} 5 \mathrm{~N} 1$ avian influenza virus using ostrich (Struthio camelus) yolk. Mol Med Rep 1: 203-209, 2008.

16. Reed LJ and Muench H: A simple method of estimating fifity percent endo-points. Am J Hyg 27: 493-797, 1938.

17. Lu L, Yu L and Kwang J: Baculovirus surface-displayed hemagglutinin of $\mathrm{H} 5 \mathrm{~N} 1$ influenza virus sustains its authentic cleavage, hemagglutination activity, and antigenicity. Biochem Biophys Res Commun 358: 404-409, 2007.

18. Bosch FX, Orlinch M, Klenk HD and Rott R: The structure of the hemagglutinin, a determinant for the pathogenicity of influenza viruses. Virology 95: 197-207, 1979.

19. Gralton J and McLaws ML: Protecting healthcare workers from pandemic influenza: N95 or surgical masks? Crit Care Med 38: 657-667, 2010.

20. Aiello AE, Murray GF, Perez V, Coulborn RM, Davis BM, Uddin M, Shay DK, Waterman SH and Monto AS: Mask use, hand hygiene, and seasonal influenza-like illness among young adults: a randomized intervention trial. J Infect Dis 201: 491-498, 2010. 